\title{
Revolutionizing Healthcare through Technology
}

\author{
Yanamandra $\mathrm{R}^{*}$ \\ Skyline University College, UAE \\ *Corresponding author: Ramakrishna Yanamandra, Skyline University College, \\ UAE, Tel: +97165441155; Email: ramakrishna@skylineuniversity.ac.ae
}

\author{
Editorial \\ Volume 2 Issue 6
}

Received Date: December 26, 2019

Published Date: December 30, 2019

DOI: $10.23880 /$ jqhe- 16000147

\section{Editorial}

Healthcare industry has been one of the fastest growing industries in the recent past. Advancements in medical research, medical technology, population growth, increasing awareness about preventive healthcare and medical insurance are among the many factors contributing towards this growth. Significant contributions from areas of healthcare industry like pharma, biotechnology, genetics and diagnostic services have also enabled this growth. Role of technology and innovative medical solutions have revolutionized the healthcare quality and improved diagnosis and patient service in many countries. Some of the diseases related to heart, brain, kidneys, lungs, liver and diabetes, considered to be life-threatening previously have become controllable and curable ones due to these technological improvements. These innovative medical technologies range from a very small device like an elbow brace to highly advanced CT scanners, artificial parts of body to three-dimensional printing.

\section{An Overview of Some of these Technologies is Presented in this Article}

Technology has influenced almost all areas of medical field. For instance, technology has enabled continuous monitoring of hearts of patients through the usage of sensors like watch, wristband and necklace. In addition to this, diagnosis and surgeries related to heart are done using advanced technologies like Left Ventricular Assist Device (LVAD), low-dose CT scan, Transcatheter Aortic Valve Replacement (TVAR), and Percutaneous Coronary Intervention (PCI). Harvard Medical School predicts the usage of more innovative upcoming cardiac technologies in future like Google Glass, Bioresorbable stents, leadless pacemaker, Value-in-valve procedure and Protein patch for heart muscle growth. Similarly, technologies like personalized vaccines, cell therapy, gene editing and microbiome treatments are the four technologies going to improve the way cancer is diagnosed and treated in future. In the same manner, several technological innovations have changed the way respiratory, gastroenterology, kidney and brain disorders are diagnosed and treated. Also, other technologies like 3D printing, big data, and cloud computing and mobile applications related to medical field have influenced storing, processing and interpreting data related to medical history of patients. Digitization of health records of patients and analysis of this data using big data analytics has improved the diagnosis and surgical processes tremendously [1-3].

Big data in healthcare has provided several benefits like reduction of medical expenses, ease of medical procedures, reduction of wastage, and improvement of overall quality of healthcare. Internet of Things (IoT) and Cloud Computing are able to information to patients at a very limited cost and ease of access. Medical practitioners are able to observe and track sleeping patterns of patients, advise them on eating habits and physical exercise using technology. Monitoring of patients from home, especially the old aged and non-movable patients without troubling them with frequent visits to clinics has been one of the greatest achievements of medical technology. Mobile apps, pacemakers and synchronization of real-time data of patients with the servers in hospitals have enabled reduction of visits by the patients. Technology has improved the communication between the patients to doctors and hospitals to patients / doctors. Another area of improvement of medical communication is interaction using video-conferencing mode. This enabled the discussions between doctors across various hospitals for improved diagnosis and accurate surgical procedures. With the help of technology, patients are able to book appointments and buy medicines online. They are also able to compare, analyse and take appropriate decisions 


\section{Journal of Quality in Health Care \& Economics}

related to their medical problems online using the information available. With the help of advanced robotics, doctors are able to perform some of the surgeries virtually without being physically present in the operation theatres. Robotics have also contributed towards minimal invasive surgeries and provided greater ease and comfort for patients. The recovery time of patients after the surgery has also significantly reduced along with expenditure related to it. Information and communication technology (ICT) links healthcare professionals with patients. Email, smartphones, telemedicine, and telemonitoring systems are all used to share information and are especially useful for more rural areas and locations with a lack of facilities and/or specialists. Some of the mobile applications have helped hard core smokers to quit smoking and receive psychological counseling through neuromodulation. Neural-bypass technology enabled reduction of partial paralysis among several patients.
The present trend of significant influence of technology is expected to grow in the next decade and take healthcare services to the next level. At the same time, hospitals and other healthcare institutions should focus on making these technologies affordable by all sections of people in the society.

\section{References}

1. New Technology Improves Heart Disease Screenings \& Treatment.

2. (2015) 5 new cardiac technologies to watch.

3. Four New Technologies That Will Change Cancer Treatment. 